Research Article

\title{
Intelligent Control System in Desert Areas Based on Photovoltaic Microgrid Power Supply
}

\author{
Jianjun $\mathrm{Li}^{1,2}$ and Gaoqing $J i \mathbb{D}^{3}$ \\ ${ }^{1}$ College of Information and Electrical Engineering, China Agricultural University, Haidian District, Beijing 100083, China \\ ${ }^{2}$ Modern Agricultural Engineering Key Laboratory, Tarim University, Alar 843300, Xinjiang, China \\ ${ }^{3}$ College of Electrical Engineering, Hebei University of Architecture, Zhangjiakou 075000, Hebei, China
}

Correspondence should be addressed to Gaoqing Ji; jgq1941@hebiace.edu.cn

Received 1 June 2021; Revised 21 June 2021; Accepted 3 July 2021; Published 20 July 2021

Academic Editor: Sang-Bing Tsai

Copyright ( $\odot 2021$ Jianjun Li and Gaoqing Ji. This is an open access article distributed under the Creative Commons Attribution License, which permits unrestricted use, distribution, and reproduction in any medium, provided the original work is properly cited.

\begin{abstract}
With the advent of the global energy crisis, the use of sustainable green energy has become more and more widespread and the utilization rate of photovoltaic industry in high-altitude desert areas is getting higher and higher. This article mainly studies the intelligent control system in desert area based on photovoltaic microgrid power supply. The system uses shielded twisted pair to transmit signals, and electrostatic interference and electromagnetic induction interference are effectively suppressed. After ensuring that the encoder feedback is correct and the motor movement direction is consistent with the command direction and the safety of the movement stroke, the PID parameters of the motor are tuned in PAMCturningpro. For the performance test of this system, the design and implementation of the test plan should be carried out according to the concurrent support target and response time target proposed in the performance analysis of the system. Concurrent users use the LoadRunner tool to create the customer test machine to simulate the concurrent access and operation of the system. In each iteration process, the test results are recorded in the same way as the benchmark test and finally the system can still achieve the expected performance when the actual concurrency reaches the limit of 5007. The results show that the intelligent control system in this paper has the advantages of low cost, convenient operation, perfect function, high precision, stable and reliable operation, etc. and has good practical value.
\end{abstract}

\section{Introduction}

The traditional photovoltaic power supply is an uncontrollable unit on the grid side, and the output disturbance directly affects the grid, so it is unable to maximize the use of solar energy. If you want to ensure a constant power output, you need to be equipped with a large capacity energy storage device, but its economy is unreasonable. However, the photovoltaic power supply system in the microgrid is controllable for the grid, which can realize the two-way flow of power and make the control more flexible.

Microgrid can not only be integrated into the large power grid but also be operated off the grid and can realize seamless switching between the two states. For the large power grid, the microgrid will be a variety of renewable energy distributed generation which is not easy to control to form a schedulable system; for users, the microgrid can meet its diverse and high reliability power demand. Therefore, this paper studies the control strategy of photovoltaic power supply system in microgrid, which has practical significance to promote the use efficiency of renewable energy and solve the problem of nonrenewable energy shortage.

In recent years, with the continuous expansion of power system capacity, the continuous improvement of voltage levels, and the increasing requirements of users for power supply reliability, people's requirements for conditional maintenance of transformers are becoming more and more urgent. Kumar PM believes that vehicles on the vehicle Internet can communicate with each other to determine the status of roads and vehicles in real time. These parameters are used to estimate the average speed and determine the best route to the destination. However, the government 
transportation department is unable to use these valuable traffic data, causing more traffic congestion and traffic accidents. To solve this problem, he proposed an effective traffic control system based on IoV technology. He applied the ant colony algorithm to each map to find the optimal path. In addition, he also proposed a traffic intensity calculation function based on fuzzy logic to model high-volume traffic. He compared the proposed IoV-based path selection algorithm with the existing Dijkstra algorithm, Kruskal algorithm, and Prim algorithm. Although his algorithm is more effective, it lacks specific research data [1]. Liu et al. believe that solar photovoltaic power generation is a promising alternative method of power supply for buildings, which dominates most urban areas. They classified the research progress of photovoltaic integrated electric energy storage technology into mechanical, electrochemical, and electric energy storage types and analyzed it from the aspects of technology, economy, and environment. In addition, based on the adopted optimization criteria, they analyzed and discussed extensive research on hybrid photovoltaic energy storage systems to improve their application prospects in buildings. Although their research content provides clear guidance for further research in related fields, it lacks specific practical steps [2]. Roy Ghatak believes that, in the context of global warming, deregulation of the energy market, and substantial load growth, the distribution network needs a complete set of strategies to maintain the reliability and efficiency of power supply. He believes that combining solar photovoltaic power generation (PV) systems and battery energy storage (BS) with distributed static compensators (DSTATCOM) is an effective and practical method to alleviate power quality and reliability problems. He proposed a comprehensive strategic model to optimize the deployment of photovoltaics, base stations, and DSTATCOM to maximize the voltage profile, reliability, and economic and ecological benefits of the network. He proposed a new type of accurate voltage distribution improvement index and network voltage distribution improvement index. He proposed the use of benefit-cost ratio and environmental benefit index to quantify economic and environmental benefits. Although his research has certain reference value, it is not innovative enough [3].

This paper establishes a cost equation based on the overall demand. The simulation results in this paper provide a reliable theoretical basis for the subsequent parameter prediction and analysis. The maintenance strategy based on the results of the status evaluation can improve the rationality of the equipment maintenance cycle through a comprehensive analysis of the equipment operating status and overcome the blindness and rigidity of the previous regular maintenance.

\section{Intelligent Control System for Detection Equipment in Desert Areas}

2.1. Photovoltaic Microgrid Power Supply. Photovoltaic panel is a kind of semiconductor device with silicon as the main material. When sunlight of appropriate intensity shines on the surface of the solar panel, the energy is absorbed by the solar panel to generate electricity. In the microgrid, the main power supply is the energy storage devices of small units with power electronic interface, such as microgas turbine, fuel cell, photovoltaic cell, small wind turbine, supercapacitor, flywheel, and battery. In this paper, the microgrid power supply is used as photovoltaic cells. It can not only reliably supply power to local load but also be integrated into large power grid. Generally, photovoltaic panels are connected through a certain series and parallel circuit to form a photovoltaic array combination [4]. The expression for manually adjusting the loss weight $\lambda^{k}$ is as follows:

$$
\begin{aligned}
\lambda^{k}(t) & =\frac{K \exp \left(\delta_{k}(t-1) / T\right)}{\sum_{t} \exp \left(\delta_{t}(t-1) / T\right)}, \\
\delta_{k}(t-1) & =\frac{l_{k}(t-1)}{l_{k}(t-2)} .
\end{aligned}
$$

Here, $\delta_{k}(\cdot)$ represents the relative decline rate of the loss of task $k, t$ is the training iteration algebra, and $T$ represents the softness factor that controls the weight of the task [5].

At sampling time $k$, the output of the discrete PID controller can be expressed as follows:

$$
u_{\mathrm{PID}}(k)=K_{c}\left\{e(k)+\frac{T}{T_{i}} \sum_{i=0}^{k} e(i)+\frac{T_{d}}{T}[e(k)-e(k-1)]\right\} \text {. }
$$

Here, $u_{\mathrm{PID}}(k)$ is the output of the PID controller at time $k$ and $e(k)=y_{\mathrm{sp}}-y(k)$ is the error at time $k[6]$.

Assume that the three parameters $K_{p}, K_{i}$, and $K_{d}$, respectively, represent the proportional, integral, and derivative gains of the PID. The range of variation of $K_{p}$ and $K_{D}$ is $\left[K_{P, \text { min }}, K_{P, \text { max }}\right]$ and $\left[K_{D \text {,min }}, K_{D \text {,max }}\right]$, which can be determined by experience or experiment [7]. Through the following linear transformation, the normalization becomes the parameters $K_{P}^{\prime}$ and $K_{d}^{\prime}$ between 0 and 1 :

$$
\begin{gathered}
K_{P}^{\prime}=\frac{\left(K_{P}-K_{P, \text { min }}\right)}{\left(K_{P, \text { max }}-K_{P, \text { min }}\right)}, \\
K_{d}^{\prime}=\frac{\left(K_{d}-K_{d, \text { min }}\right)}{\left(K_{d, \text { max }}-K_{d, \text { min }}\right)} .
\end{gathered}
$$

The integration time constant is determined by the differential time constant:

$$
T_{i}=\alpha T_{d}
$$

The integral gain is calculated by the following formula:

$$
K_{i}=\frac{K_{p}}{\alpha T_{d}}=\frac{K_{p}^{2}}{\alpha K_{d}} .
$$

When the DMA output completion interrupt is generated, that is, the ADC has completed the data sampling of 1 signal cycle, it first extracts the discrete data of ten channels from the DMA storage area [8]. Here is a single-phase AC signal voltage signal and current signal as an example, 
assuming that the discrete voltage signal and discrete current signal are, respectively,

$$
\begin{aligned}
U_{n}[n] & =\left\{u_{0}, u_{1}, u_{2}, \ldots, u_{n}\right\}, \\
I_{n}[n] & =\left\{i_{0}, i_{1}, i_{2}, \ldots, i_{n}\right\} .
\end{aligned}
$$

First, subtract the quantized values $u_{D}$ and $i_{D}$ of the DC part of the signal and get the new signal as

$$
\begin{aligned}
U_{n}^{\prime}[n] & =\left\{u_{0}-u_{D}, u_{1}-u_{D}, u_{2}-u_{D}, \ldots, u_{n}-u_{D}\right\}=\left\{u_{0}^{\prime}, u_{1}^{\prime}, u_{2}^{\prime}, \ldots, u_{n}^{\prime}\right\}, \\
I_{n}^{\prime}[n] & =\left\{i_{0}-i_{D}, i_{1}-i_{D}, i_{2}-i_{D}, \ldots, i_{n}-i_{D}\right\}=\left\{i_{0}^{\prime}, i_{1}^{\prime}, i_{2}^{\prime}, \ldots, i_{n}^{\prime}\right\} .
\end{aligned}
$$

The valid values of ADC pin signal are $U_{\text {rms }}$ and $I_{\text {rms }}[9]$.

$$
\begin{gathered}
U_{\mathrm{rms}}=\sqrt{\frac{1}{n} \sum_{j=0}^{n-1}\left(U_{n}^{\prime}[j]\right)^{2},} \\
I_{\mathrm{rms}}=\sqrt{\frac{1}{n} \sum_{j=0}^{n-1}\left(I_{n}^{\prime}[j]\right)^{2} .}
\end{gathered}
$$

The physical object of the multifunction measurement and control box is shown in Figure 1. The signal acquisition circuit is mainly responsible for the acquisition of the voltage and current signals of the electrical circuit, so that the main control chip can perform analysis and calculation. The main function of the clock circuit module is to provide an accurate time for the system to facilitate accurate real-time monitoring and measurement. Because the multifunction measurement and control box continuously collect various electricity consumption circuits in a polling manner, the obtained data must be stored in a relatively large space. The main purpose of the electrical parameter display module is to display the electrical parameters collected and calculated by the multifunction measurement and control box to facilitate the observation of data $[10,11]$.

The voltage sampling circuit is shown in Figure 2. If the voltage at the input end of the transformer is $220 \mathrm{~V}$, because the resistance of the primary side is connected in series by a sliding rheostat and two 100k parallel resistors, when the potentiometer adjusts when the resistance value of the primary side becomes $55 \mathrm{k}$, the current of the primary side of the voltage transformer can reach $4 \mathrm{~mA}$ [12]. The masterslave control strategy is mainly used in islanding operation of microgrid. When grid connected, the overall capacity of the microgrid is smaller than that of the grid, the grid can stabilize the frequency of the system, and the microgrid does not need to adjust the frequency and only needs to output the specified active power and reactive power; when the islanding mode is running, the master control unit of the master-slave control system adopts V/F control to maintain the constant voltage and frequency of the system.

\subsection{Intelligent Control System for Testing Equipment in Desert} Areas. In the desert irrigation microgrid system, the hybrid energy storage device controlled by the energy management system can adjust the energy of the system according to the needs of the system at different times. When the load is in urgent need of power supplementation and the wind and photovoltaic power generation units cannot meet the power output, due to the high power density and fast response speed of the supercapacitor, the supercapacitor can be controlled to give priority to the load by adjusting the converter [13]. When the load in the microgrid changes, the main control source first automatically adjusts the output current according to the load change to make the output power increase or decrease; at the same time, it detects and calculates the change of its own power and adjusts the output value of other subordinate systems according to the available capacity of the existing generation unit to make their output power change; when the output power of other slave systems increases, the output of the master control source decreases accordingly, so that the master control source always has enough capacity to adjust the change of instantaneous power $[14,15]$.

The physical structure of the system is shown in Figure 3. The physical topology of the system is designed according to the actual deployment situation. Based on the internal resource status of a power company, the B/S-based Web service distributed architecture is specifically adopted, including system end users such as power equipment users and maintenance personnel (collectively referred to as equipment operation monitoring personnel); data management personnel use the business communication network and the system Web server to establish communication to realize the status view of the power equipment, maintenance, and fault management operations. At the same time, the APP terminal in the system uses the mobile terminal interactive interface to realize data query. System server includes web server, data server, and monitoring database server, and all service resources are stored in Aliyun space [16]. The monitoring data of each power equipment is saved in the SCADA system database, and the data saved in the SCADA system database is structured data [17].

As the output power of photovoltaic power generation and wind power generation fluctuates with the changes of light intensity, temperature, wind speed, and other factors, it is impossible to control its maximum power output, so this kind of power supply is uncontrollable. It belongs to controllable power supply (such as flywheel), energy storage system, etc. Because the controllable power supply cannot use renewable energy, the output power of microgrid should 


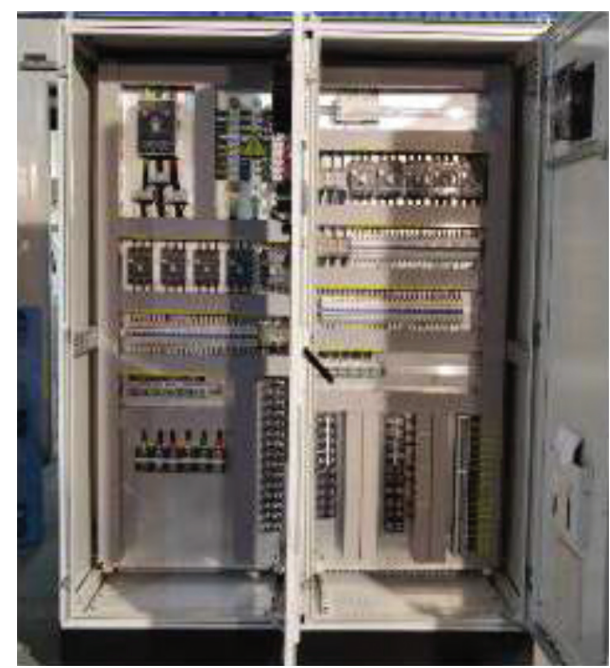

FIgURE 1: Multifunctional measurement and control box in kind (picture from http://alturl.com/d3noh).

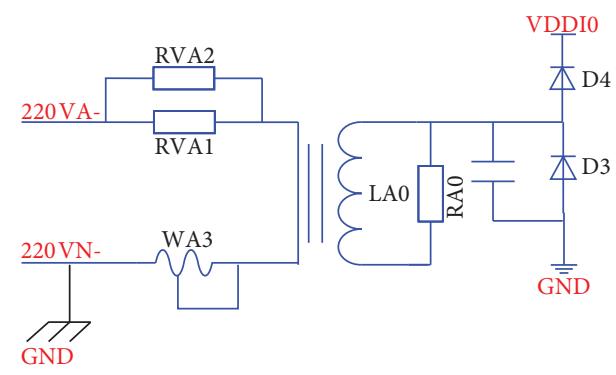

FIgURE 2: Circuit for voltage sampling.

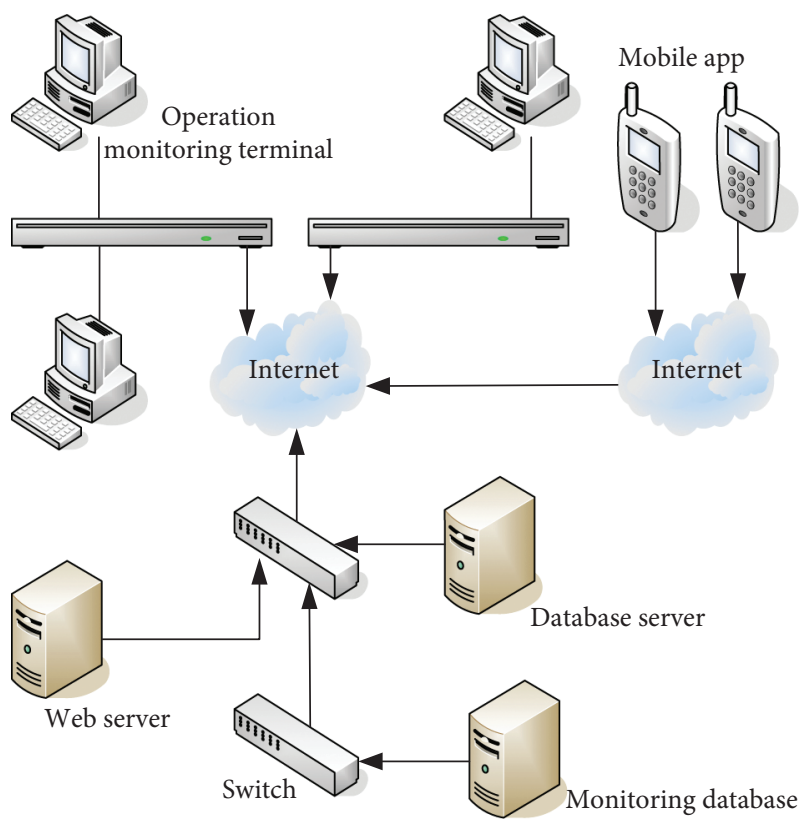

Figure 3: System's physical structure.

be reduced as much as possible to make full use of renewable energy on the premise of ensuring the qualified power quality of microgrid $[18,19]$.
The staff here use the master control computer to complete various required operations and are equipped with a printer, which can print relevant reports at any time, and can display the data of each collection point in real time through the LCD. The concentrator summarizes the information uploaded by the various measurement and control points of the whole school and uploads it to the server through the local area network. At the same time, the various commands downloaded from the master control computer are transmitted to the metering control cabinet through Ethernet/GSM, and the master control computer and the acquisition control unit completed communication and data transmission [20].

Using transformer isolation on the one hand improves the security of the system and, on the other hand, ensures that the photovoltaic power generation system will not inject DC components into the grid, effectively preventing the saturation of distribution transformers in the grid. However, the power frequency transformer is bulky and heavy, accounting for about $50 \%$ of the total weight, which makes the overall size of the inverter larger. In addition, the power frequency transformer increases the system loss, noise, cost, and the difficulty of transportation and installation [21]. As the training progresses, some auxiliary tasks no longer have a positive impact on the main task after reaching the highest performance. In moment distance or low baud rate wave data transmission, terminal matching resistance is not required; that is, generally below 300 meters, 19200 bps does not need terminal matching resistance. The terminal matching resistor is installed at the two end points of the RS485 transmission network and connected in parallel between the $\mathrm{AB}$ pins [22].

\section{Testing Equipment Intelligent Control System Experiment}

3.1. Development Platform. The local gateway hardware development platform is equipped with Samsung S5PV210 processor, which uses ARMCortexTM-A8 architecture and ARMV7 instruction set. For software development based on the Android operating system, Linux device drivers should first be written as needed. The device driver completes the initialization of the hardware and other operations. After the device driver file is successfully compiled, it is suffixed with ko. After the writing is completed, the device driver file is loaded in the form of a kernel module. In the Linux kernel, and make device file in the Linux kernel $[23,24]$.

3.2. Antijamming Design. Using shielded twisted pair to transmit signals, electrostatic interference and electromagnetic induction interference are effectively suppressed. The signal wires between the power supply module and the monitoring unit are all twisted-pair shielded wires. In addition, a shielding net can be added between the power supply module and the monitoring module to reduce the radiation interference of the power supply module to the monitoring unit [25]. 
3.3. PMU Test. At room temperature, use Datalog to connect five $2 \mathrm{~kW}$ low-power single-phase inverters, and use DC source Chroma6260 to simulate photovoltaic modules to provide DC input to the inverter, and the AC output of the inverter is directly connected to the laboratory grid [26].

3.4. Motor Trajectory Planning Experiment. After ensuring that the encoder feedback is correct, the motor movement direction is consistent with the command direction and the safety of the movement stroke, the PID parameters of the motor are tuned in PAMCturningpro. Set the gain $K_{p}$, the differential constant $K_{d}$, the integral constant $K_{i}$, the velocity feedforward $K_{\mathrm{vff}}$, and the acceleration feedforward $K_{\text {aff }}$ to zero. In the step response test, adjust the parameter values of $K_{p}, K_{d}$, and $K_{i}$, so that the motor has a fast response to the step response and lower damping and steady-state error.

3.5. System Debugging. When collecting the working voltage and current of the photovoltaic power station, due to the interference of the environment and system circuit noise, and because the Hall sensor for current collection is very sensitive, the monitored data and the actual data will have a certain degree of deviation. Photovoltaic inverter works in high-frequency mode, its high switching frequency will produce harmonic pollution to the power grid, and its output current will produce serious harmonic pollution to the power grid. In microgrid system, photovoltaic power interface inverter is an important connection module between microgrid and high voltage grid, which can convert DC power from photovoltaic power into AC power or provide energy for local load. In order to eliminate the interference noise on the monitoring signal, an RC low-pass filter is added to the appropriate part of the circuit to effectively suppress high-frequency differential mode. The signal interference is evenly distributed in the layout of the circuit and the components [27].

3.6. Performance Benchmarking. For the performance test of this system, the design and implementation of the test plan should be carried out according to the concurrent support target and response time target proposed in the performance analysis of the system. Concurrent users use the LoadRunner tool to create in the customer test machine to simulate the concurrent access and operation of the system. At the same time, the performance monitoring points of the system performance test select 4 functions that can represent the actual performance of the system as specific test scenarios, which are the parameter setting and data collection processing in the monitoring data acquisition module of the system, and the Web operation monitoring terminal module which are the parameter setting and data collection and processing in the system monitoring data acquisition module, the Web operation monitoring terminal module, the monitoring data analysis and algorithm processing functions, and the APP management function module [28].

\section{Results and Discussion}

4.1. Performance Test Results. In the test, the limit test is also tested based on the LoadRunner tool. The specific test method of the limit test is to select some representative functions from the functional system of the system, use the test case scripts of these functions, and use the LoadRunner tool as a concurrent simulation tool to continuously iteratively boost the server side of the system in the test environment. Check the actual performance of the system. The system limit test results are shown in Table 1. Through regression iteration, the performance test is performed on each different limit point. With the continuous increase of concurrency, the number of regression iterations also increases. In each iteration process, the test results are recorded in the same way as the benchmark test, and finally the system can still achieve the expected performance when the actual concurrency reaches the limit of 5007.

The changes of various data of photovoltaic modules in a day over time are shown in Figure 4. The output power of the photovoltaic module is proportional to the daily ambient temperature and the intensity of the light irradiated on the surface of the photovoltaic module; that is, when the temperature and light intensity are large, the output power of the photovoltaic module is also large; when the temperature and light intensity gradually change when it is small, the output power also decreases.

The comparison of the data collected by the monitoring system and the actual value is shown in Table 2. The absolute value of the error between the voltage and current measured value and the actual value of the photovoltaic module collected by the monitoring system is less than $3 \%$, and the absolute value of the error becomes smaller as the voltage and current value gradually increase.

The comparison of the test results is shown in Table 3. Through the ledger record and real-time collection of each possible fault status of the transformer, with the ESN intelligent analysis method, the analysis result can be automatically given by the detection system, and the intelligent detection of the status of the transformer, the core power equipment of the substation, is realized. It can be seen from the detection results that the accuracy of the intelligent detection system in this paper can reach $100 \%$, which is far higher than the accuracy of manual detection; the detection speed in this paper is 1.25 minutes, while the manual detection speed is 35.58 minutes. Therefore, the intelligent detection system in this article has a very good application effect.

4.2. Intelligent Test Results. The power generation of photovoltaic modules in a day under different weather conditions is shown in Figure 5. It can be seen from the curve that, under good sunshine conditions, the power generation increases rapidly, which is significantly better than that under insufficient sunshine conditions. Under insufficient sunshine weather, the cumulative power generation of photovoltaic modules increases slowly and the value is small [29]. 
TABLe 1: System limit test results.

\begin{tabular}{lcc}
\hline Expected concurrency & Actual concurrency & Response time performance index \\
\hline 100 & 102 & Maximum: $1.05 \mathrm{~s}$ \\
500 & 501 & Maximum: $1.24 \mathrm{~s}$ \\
1000 & 1004 & Maximum: $1.36 \mathrm{~s}$ \\
2000 & 2009 & Maximum: $1.48 \mathrm{~s}$ \\
3000 & 3017 & Maximum: $1.57 \mathrm{~s}$ \\
5000 & 5007 & Maximum: $1.69 \mathrm{~s}$ \\
\hline
\end{tabular}

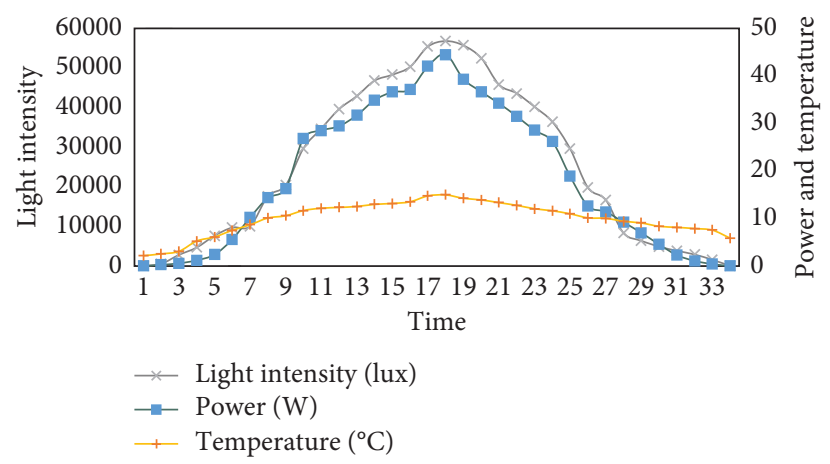

Figure 4: Variations of various data of photovoltaic modules over time in a day.

TABLE 2: Comparison of data collected by the monitoring system and actual values.

\begin{tabular}{|c|c|c|c|c|c|c|}
\hline Time & Voltage measurement (V) & Actual value & Error & Current measurement (A) & Actual value & Error \\
\hline $7: 20$ & 8.945 & 9.125 & $-1.97 \%$ & 0.096 & 0.098 & $-2.04 \%$ \\
\hline 8:00 & 16.472 & 16.771 & $-1.78 \%$ & 0.457 & 0.469 & $-2.56 \%$ \\
\hline 9:00 & 17.521 & 17.835 & $-1.76 \%$ & 0.839 & 0.823 & $1.94 \%$ \\
\hline 10:00 & 18.948 & 18.659 & $1.55 \%$ & 1.587 & 1.614 & $-1.67 \%$ \\
\hline 11:00 & 19.112 & 19.397 & $-1.47 \%$ & 1.827 & 1.856 & $-1.56 \%$ \\
\hline $12: 00$ & 19.876 & 20.142 & $-1.32 \%$ & 2.193 & 2.225 & $-1.44 \%$ \\
\hline 13:00 & 19.236 & 19.517 & $-1.44 \%$ & 2.015 & 1.983 & $1.61 \%$ \\
\hline 14:00 & 18.951 & 18.669 & $1.51 \%$ & 1.583 & 1.608 & $-1.55 \%$ \\
\hline 15:00 & 17.509 & 17.208 & $1.75 \%$ & 0.849 & 0.863 & $-1.62 \%$ \\
\hline $16: 00$ & 15.081 & 15.370 & $-1.88 \%$ & 0.581 & 0.592 & $-1.86 \%$ \\
\hline 17:00 & 12.308 & 12.566 & $-2.05 \%$ & 0.087 & 0.089 & $-2.25 \%$ \\
\hline
\end{tabular}

TABLE 3: Comparison of test results.

\begin{tabular}{lccc}
\hline Test method & Number of test groups & Detection accuracy (\%) & Detection speed \\
\hline Manual detection & 20 & 95 & 35.58 minutes \\
Intelligent detection of the system & 20 & 100 & 1.25 minutes \\
\hline
\end{tabular}

The voltage test result is shown in Figure 6. Because the accuracy of the voltage measurement is required to be within $0.5 \%$, because the peak voltage is within the $0-500 \mathrm{~V}$ measurement range, the maximum absolute error is less than $2.5 \mathrm{~V}$, and the Fluke5502 A calibrator used in this experiment outputs the effective value of the voltage, and the maximum absolute error should be less than $1.77 \mathrm{~V}$. It can be observed from the figure that the maximum absolute error of current measurement is $1.5 \mathrm{~V}$, and the relative error of each group of data is less than $0.5 \%$.

In this system, the protocol to realize certain functions needs to be written according to the frame format of table. When the upper computer issues commands, it is necessary to transfer information to its command receiver according to the defined protocol into a series of binary codes. When the other party receives the information, it first decodes it according to the protocol, then performs corresponding operation according to the content of the information, and transmits the corresponding response frame to the sender of the command in the same way, and then the requesting party of the command interprets the corresponding answer frame, thus completing the whole process. The voltage of DC bus without energy storage device and energy storage device in microgrid is shown in Figure 7. It can be seen from the figure analysis that when the microgrid system does not have energy storage device, the output fluctuates due to the weather conditions of the power generation unit, which leads to the change of DC bus voltage. With 


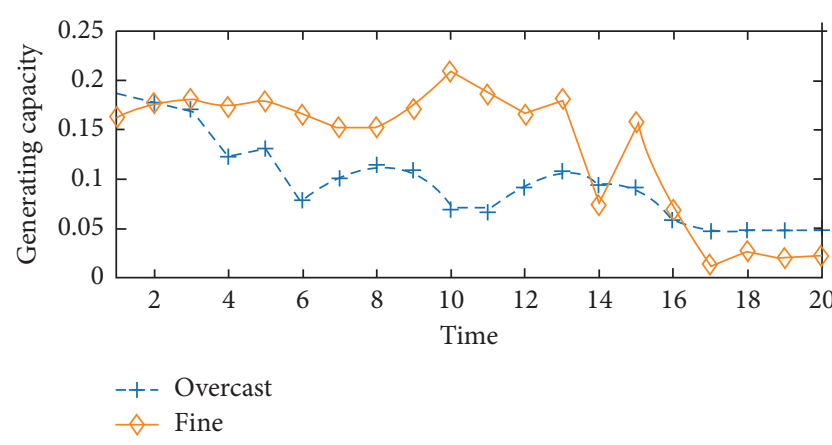

Figure 5: The power generation of photovoltaic modules in a day under different weather.

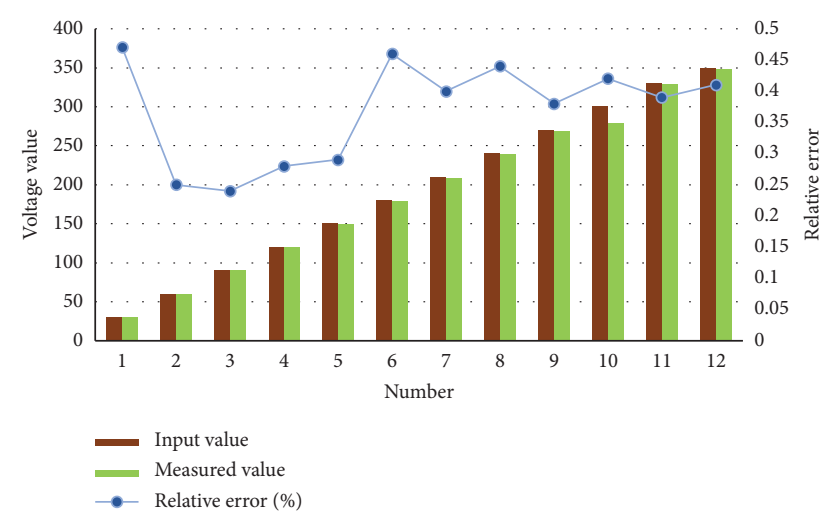

Figure 6: Voltage test results.

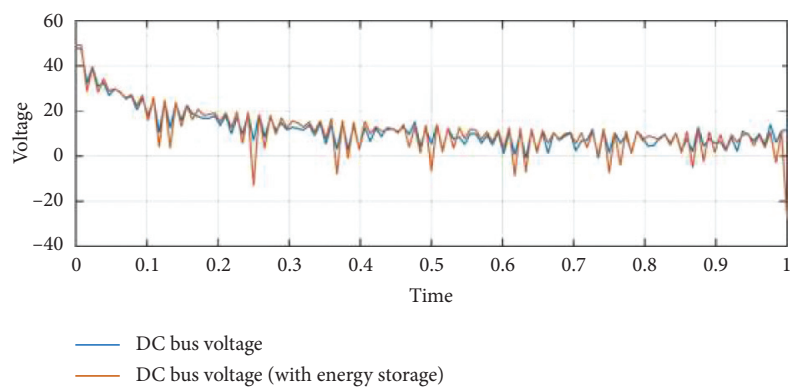

FIGURE 7: The DC bus voltage of the microgrid without and with energy storage devices.

presynchronization control, the frequency fluctuation of the microgrid can be stable within $\pm 0.5 \mathrm{~Hz}$, and the voltage amplitude fluctuation can be stable within $\pm 5 \%$ of its rated voltage. All meet the requirements of stable operation of microgrid. Therefore, the simulation results verify the correctness and effectiveness of the presynchronization switching control strategy.

The working status of the converter on the day to be verified is shown in Table 4. It can be seen from the table that the AC and DC converters were switched on and off for 5 times on that day, and the total grid-connected time on the DC side was 5 hours and 26 minutes. Obviously, the traditional coordination strategy of the green energy building power supply system applied the traditional coordination strategy to the number of impacts on the AC measurement
TABLE 4: Working status of the converter on the day to be verified.

\begin{tabular}{lc}
\hline Time & Converter status \\
\hline $9: 15$ & Resection work \\
11:30 & Work cut \\
13:21 & Resection work \\
16:30 & Work cut \\
18:00 & Resection work \\
Total switching times & 5 times \\
Total working hours & 5 hours 26 minutes \\
\hline
\end{tabular}

and even the AC power grid. Energy pollution takes a long time. At the same time, the energy storage system is in a constant current shutdown state within 5 hours and 26 minutes. Part of the electrical energy generated by photovoltaics is transmitted to the AC measurement and feedback to the AC grid, which causes a waste of photovoltaic output power during the day. Due to the active switching based on prediction, the charge of the battery can be adjusted in advance, so that the number of switching on and off during the day on the day to be verified is reduced from 5 times to once at 18:00, and the grid-connected time of the DC side is 0 . It greatly increases the utilization of photovoltaic power on the DC side and at the same time greatly reduces the negative impact of green energy on the large AC grid, including the shock caused by 4 switching times and the power quality impact of more than 5 hours.

4.3. System Function Test Results. The main interface response time of the Web service is shown in Table 5. Since the Web service and the distributed cluster are separated during the system design, it can get a faster response when requesting the Web service. The average response time is $460 \mathrm{~ms}$ in the case of 10 concurrencies, and the average response time is $1012 \mathrm{~ms}$ in the case of 100 concurrencies.

The comparison between the predicted instability trajectory and the actual instability trajectory is shown in Figure 8. The prediction curve can better fit the actual curve. Through statistics on the prediction results, it can be known that when the time window length is $200-300 \mathrm{~ms}$, the normalized mean square error of the predicted stable trajectory can be controlled within 0.03 , and the unstable trajectory can be predicted. The normalized mean square error can be controlled within 0.04 . Under a single disturbance, MPPT tracking speed is very fast (about $7 \mathrm{~ms}$ ) in the start-up stage, and the dynamic performance is very good. The PV current IPV, PV voltage VPV, and photovoltaic power PPV can also achieve stable state after about $7 \mathrm{~ms}$. The system tracks the MPP at the maximum power point with high precision, and there is no oscillation near the maximum power point; that is, the steady-state performance is quite good.

The environmental parameter test results are shown in Table 6. The test results show that the information-based intelligent power supply equipment can accurately measure the normal ambient air pressure value. In view of laboratory conditions, it cannot be measured in multiple air pressure ranges. According to the above table, the maximum error of 
TABLE 5: Web service main interface response time.

\begin{tabular}{lccc}
\hline Concurrency & Minimum (ms) & Maximum (ms) & Average (ms) \\
\hline 10 & 29 & 1185 & 460 \\
100 & 60 & 8458 & 1012 \\
\hline
\end{tabular}

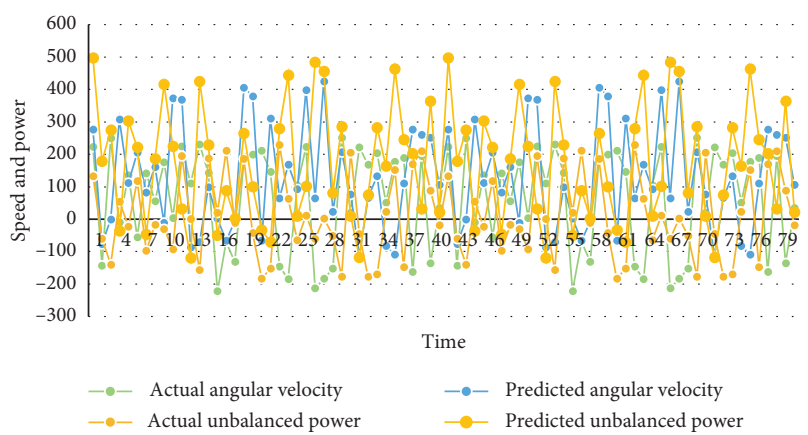

FIgURE 8: Comparison of predicted instability trajectory and actual instability trajectory.

TABLE 6: Environmental parameter test results.

\begin{tabular}{cccc}
\hline Type of test & Standard value & Measurements & Error \\
\hline \multirow{5}{*}{ Temperature } & $27.0^{\circ} \mathrm{C}$ & $27.5^{\circ} \mathrm{C}$ & $0.5^{\circ} \mathrm{C}$ \\
& $30.5^{\circ} \mathrm{C}$ & $31.2^{\circ} \mathrm{C}$ & $0.7^{\circ} \mathrm{C}$ \\
& $40.8^{\circ} \mathrm{C}$ & $41.4^{\circ} \mathrm{C}$ & $0.6^{\circ} \mathrm{C}$ \\
& $48.6^{\circ} \mathrm{C}$ & $49.2^{\circ} \mathrm{C}$ & $0.6^{\circ} \mathrm{C}$ \\
& $55.6^{\circ} \mathrm{C}$ & $56.3^{\circ} \mathrm{C}$ & $0.7^{\circ} \mathrm{C}$ \\
\hline \multirow{5}{*}{ Humidity } & $26.8 \% \mathrm{RH}$ & $24.3 \% \mathrm{RH}$ & $2.5 \% \mathrm{RH}$ \\
& $32.5 \% \mathrm{RH}$ & $29.7 \% \mathrm{RH}$ & $2.8 \% \mathrm{RH}$ \\
& $53.9 \% \mathrm{RH}$ & $50.6 \% \mathrm{RH}$ & $3.3 \% \mathrm{RH}$ \\
& $70.5 \% \mathrm{RH}$ & $68.7 \% \mathrm{RH}$ & $1.8 \% \mathrm{RH}$ \\
\hline
\end{tabular}

temperature measurement is $0.7^{\circ} \mathrm{C}$, and the maximum error of humidity measurement is $3.3 \% \mathrm{RH}$. For humidity measurement performance indicators, therefore, it can be considered that the temperature and humidity measurement results of information-based intelligent power supply equipment basically meet the system performance indicators.

\section{Conclusions}

Based on PSCAD platform, the paper designs the AC photovoltaic microgrid with peer control and the optical storage combined AC microgrid with master-slave control. The improved control strategy realizes the stable output of DC voltage and power of photovoltaic inverter in microgrid under networking mode. The output power of photovoltaic inverter in islanding mode can change with the change of load in real time, providing sustainable and reliable power, and meeting the requirements of stable operation of microgrid. The function modules include monitoring data collection, web operation monitoring end, APP end, and data analysis and prediction. In the design of the system, the professional metering chip ATT7022 is used to complete the real-time monitoring of the power information. The control process of microgrid transition from grid connected to off grid is relatively simple. When grid is connected, PQ control is still adopted. Through the final proofreading, the actual measurement results of the system can reach $0.5 \mathrm{~s}$. It can protect the normal use of reasonable electrical appliances and eliminate the potential safety hazard caused by the use of malignant load. According to the characteristics of power grid dispatching and software, the system can achieve the optimal utilization of system performance resources on the premise of ensuring the safe operation of power grid.

\section{Data Availability}

No data were used to support this study.

\section{Conflicts of Interest}

The authors declare no conflicts of interest.

\section{Acknowledgments}

This work was supported by Open Project of the Key Laboratory of Modern Agricultural Engineering of Tarim University (TDNG20180102) and Basic Scientific Research Project in Hebei Province (No. 2021QNJS13).

\section{References}

[1] P. M. Kumar, G. U. Devi, G. Manogaran, R. Sundarasekar, N. Chilamkurti, and R. Varatharajan, "Ant colony optimization algorithm with internet of vehicles for intelligent traffic control system," Computer Networks, vol. 144, no. 10, pp. 154-162, 2018.

[2] J. Liu, X. Chen, S. Cao, and H. Yang, "Overview on hybrid solar photovoltaic-electrical energy storage technologies for power supply to buildings," Energy Conversion and Management, vol. 187, no. 5, pp. 103-121, 2019.

[3] S. Roy Ghatak, S. SaNnIgrahi, and P. Acharjee, "Optimised planning of distribution network with photovoltaic system, battery storage, and DSTATCOM," IET Renewable Power Generation, vol. 12, no. 15, pp. 1823-1832, 2018.

[4] P.-H. Kuo, O.-Y. Wong, C.-K. Tzeng et al., "Improved charge pump design and ex vivo experimental validation of CMOS 256-pixel photovoltaic-powered subretinal prosthetic chip," IEEE Transactions on Biomedical Engineering, vol. 67, no. 5, pp. 1490-1504, 2020.

[5] Q. Cheng and K. Zhang, "Design of temperature intelligent PID control system based on genetic algorithm," Journal of Shenyang University of Technology, vol. 40, no. 4, pp. 459-463, 2018.

[6] L. A. Nguimdo and C. Kum, "Optimization and sizing of a stand-alone photovoltaic system and assessment of random load fluctuation on power supply," Energy and Power Engineering, vol. 12, no. 1, pp. 28-43, 2020.

[7] Y. V. Daus, V. Kharchenko, I. V. Yudaev, D. A. Desyatnichenko, and G. V. Stepanchuk, "Improving the efficiency of the power supply to agricultural facilities by means of roof-top photovoltaic installations," Applied Solar Energy, vol. 56, no. 3, pp. 207-211, 2020.

[8] A. M. Mirzabaev, V. P. Kanonerov, T. A. Makhkamov, O. R. Sytdykov, and S. M. Mirzabekov, "Photovoltaic power supply unit for the basic stations of cellular companies," Applied Solar Energy, vol. 54, no. 3, pp. 224-226, 2018. 
[9] A. N. Balalaev, S. V. Korkina, E. M. Plokhov, and A. Y. Polovinkina, "An energy-efficiency assessment of a railroad passenger-car power-supply system using wind generators and photovoltaic panels," Russian Electrical Engineering, vol. 91, no. 3, pp. 195-198, 2020.

[10] P. Sharma, M. Kolhe, and A. Sharma, "Economic performance assessment of building integrated photovoltaic system with battery energy storage under grid constraints," Renewable Energy, vol. 145, no. 1, pp. 1901-1909, 2020.

[11] T. Kato, Y. Kimpara, Y. Tamakoshi, M. Kurimoto, T. Funabashi, and S. Sugimoto, "An experimental study on dual P-f droop control of photovoltaic power generation for supporting grid frequency regulation," IFAC-PapersOnLine, vol. 51, no. 28 , pp. 622-627, 2018.

[12] S. Sadeghi and I. B. Askari, "Prefeasibility techno-economic assessment of a hybrid power plant with photovoltaic, fuel cell and compressed air energy storage (CAES)," Energy, vol. 168, no. 2, pp. 409-424, 2019.

[13] C. Tan, C. He, W. Tang, P. Kovalsky, J. Fletcher, and T. D. Waite, "Integration of photovoltaic energy supply with membrane capacitive deionization (MCDI) for salt removal from brackish waters," Water Research, vol. 147, no. 12, pp. 276-286, 2018.

[14] A. Mohammedi, D. Rekioua, T. Rekioua, and N. E. Mebarki, "Comparative assessment for the feasibility of storage bank in small scale power photovoltaic pumping system for building application," Energy Conversion and Management, vol. 172, no. 9, pp. 579-587, 2018.

[15] J. Zhou, S. Tsianikas, D. P. Birnie, and D. W. Coit, "Economic and resilience benefit analysis of incorporating battery storage to photovoltaic array generation," Renewable Energy, vol. 135, no. 5, pp. 652-662, 2019.

[16] X. Wang, S. Liu, R. Wang et al., "Research on dynamic characteristics and stability of MMC photovoltaic grid-connected system based on rotational synchronous generator model," Electric Power Systems Research, vol. 173, no. 8, pp. 183-192, 2019.

[17] H. G. Boneya, W. Li, P. Chao, and S. Peng, "A comprehensive LVRT strategy of two-stage photovoltaic systems under balanced and unbalanced faults," International Journal of Electrical Power \& Energy Systems, vol. 103, no. 12, pp. 288-301, 2018.

[18] M. Nobuhiro, N. Yorino, Y. Shimamura, Y. Tanioka, Y. Sasaki, and Y. Zoka, "Study of the operational strategy of home energy management system with photovoltaic power generation and storage battery," IEEJ Transactions on Power and Energy, vol. 139, no. 4, pp. 234-239, 2019.

[19] J. Yang, Y. Cheng, J. Jie, Z. Du, Z. Shi, and J. Han, "The impact of indoor air distributions on the thermal performance of a single layer semi-transparent photovoltaic facade," Building Simulation, vol. 12, no. 1, pp. 69-77, 2019.

[20] J. Black, A. Hoffman, T. Hong, J. Roberts, and P. Wang, "Weather data for energy analytics: from modeling outages and reliability indices to simulating distributed photovoltaic fleets," IEEE Power and Energy Magazine, vol. 16, no. 3, pp. 43-53, 2018.

[21] A. L. Bukar, C. W. Tan, and K. Y. Lau, "Optimal sizing of an autonomous photovoltaic/wind/battery/diesel generator microgrid using grasshopper optimization algorithm," Solar Energy, vol. 188, no. 8, pp. 685-696, 2019.

[22] Y.-M. Saint-Drenan, S. Vogt, S. Killinger, J. M. Bright, R. Fritz, and R. Potthast, "Bayesian parameterisation of a regional photovoltaic model-application to forecasting," Solar Energy, vol. 188, no. 8, pp. 760-774, 2019.
[23] A. Saxena, S. Deshmukh, S. Nirali, and S. Wani, "Laboratory based experimental investigation of photovoltaic (PV) thermo-control with water and its proposed real-time implementation," Renewable Energy, vol. 115, no. 1, pp. 128-138, 2018.

[24] G. de Freitas Viscondi and S. N. Alves-Souza, "A systematic literature review on big data for solar photovoltaic electricity generation forecasting," Sustainable Energy Technologies and Assessments, vol. 31, no. 2, pp. 54-63, 2019.

[25] M. Q. Duong, N. T. N. Tran, G. N. Sava, and V. Tanasiev, "Design, performance and economic efficiency analysis of the photovoltaic rooftop system," Revue Roumaine Des Sciences Techniques, vol. 64, no. 3, pp. 229-234, 2019.

[26] P. Chao, W. Li, S. Peng et al., "A unified modeling method of photovoltaic generation systems under balanced and unbalanced voltage dips," IEEE Transactions on Sustainable Energy, vol. 10, no. 4, pp. 1764-1774, 2019.

[27] S.-B. Tsai, J. Yu, L. Ma et al., "A study on solving the production process problems of the photovoltaic cell industry," Renewable and Sustainable Energy Reviews, vol. 82, pp. 3546-3553, 2018.

[28] S.-B. Tsai, "Using the DEMATEL model to explore the job satisfaction of research and development professionals in China's photovoltaic cell industry," Renewable and Sustainable Energy Reviews, vol. 81, pp. 62-68, 2018.

[29] V. Puri, S. Jha, R. Kumar et al., "A hybrid artificial intelligence and internet of things model for generation of renewable resource of energy," IEEE Access, vol. 7, pp. 111181-111191, 2019. 